\title{
Pedofilia, transtorno bipolar e dependência de álcool e opioides
}

\author{
Paedophilia, bipolar disorder and alcohol and opioid dependence
}

Vanessa Fabiane Machado Gomes Marsden

\section{RESUMO}

Diversos estudos investigaram a relação entre psicopatologia e parafilias, especialmente

\section{Palavras-chave}

Pedofilia, transtorno bipolar, dependência de heroína, transtornos relacionados ao uso de álcool. pedofilia. Transtornos de humor e ansiedade, seguidos de transtornos relacionados ao uso de substâncias, são as comorbidades mais prevalentes em pacientes com parafilias. Apresentou-se o caso de um paciente em tratamento para dependência de substâncias (álcool e heroína), transtorno bipolar e pedofilia. É importante frisar que poucos casos relatando comorbidades como essas foram descritos na literatura.

\section{Keywords}

Pedophilia, bipolar disorder, heroin dependence, alcohol-related disorders.

\begin{abstract}
Many studies have investigated the relationship between psychopathology and paraphilias, specifically paedophilia. Mood disorders, anxiety disorders, followed by substance use were the most prevalent disorders comorbid in these patients. We present the case of a patient in treatment for substance misuse (alcohol and heroin), bipolar disorder and paedophilia. To our knowledge few cases were reported describing cases of comorbidity such as this.
\end{abstract}

\section{INTRODUÇÃO}

Diversos estudos investigaram a relação entre psicopatologia e parafilias, especialmente pedofilia, ${ }^{1,2}$, e alguns sugerem que agressores sexuais possuem déficits em processos cognitivos e que esses são sintomas fundamentais de grave psicopatologia e transtornos de personalidade1. Transtornos de humor, especialmente distimia, são os mais prevalentes em comorbidade com parafilias, seguidos de transtornos de ansiedade e transtornos relacionados ao uso de substâncias ${ }^{2,3}$.
Esses achados sugerem que indivíduos com parafilias manifestam grave psicopatologia e desvios sexuais.

Em um estudo realizado em 1999, Raymond descreveu que $93 \%$ de um grupo de agressores pedófilos preenchiam critérios para algum diagnóstico do eixo I, além de pedofilia durante o curso de suas vidas 4 . Transtornos de humor e ansiedade foram novamente os achados mais comuns naquela população. Cinquenta e um por cento preenchiam critérios para dependência de álcool e 44\% para transtornos relacionados ao uso de outras substâncias. Esses achados

1 Universidade do Porto, Faculdade de Medicina, Portugal.

Recebido em

2/3/2009

Aprovado em

$21 / 5 / 2009$
Centro de Atendimento à Toxicodependência (CAT) de Vila Real Rua Dr. Manuel Cardona, Centro de Saúde, 1, CAT de Vila Real 5000-558 - Vila Real, Portugal 
revelam que existem diversas comorbidades em agressores sexuais e que a relação entre esses transtornos e o subtipo de agressão é incerta.

O objetivo do presente artigo é descrever o caso de um paciente com dependência química de opioides e álcool que apresenta concomitantemente transtorno bipolar e fantasias pedófilas e discutir a relação entre essas comorbidades de acordo com achados da literatura.

\section{CASO CLÍNICO}

O paciente é um homem de 28 anos, filho mais velho de pai alcoólico em uma frataria de 4, em acompanhamento para dependência química desde 2006. Ele iniciou o consumo de cannabis aos 17 anos, de heroína aos 18 e consumo endovenoso (EV) aos 23, apresentando consumo de 1 a 2 gramas de heroína EV/dia e álcool (10,2 a 13,6 unidades/dia). O paciente preenchia critérios diagnósticos para dependência química tanto de heroína quanto de álcool. O consumo de álcool era caracterizado pela ingestão de fermentados (vinho), culturalmente típico na sociedade portuguesa. Desde a adolescência o paciente apresentava comportamentos disruptivos, sendo cunhado como "difícil" por familiares, o que o levou a emigrar ao final da adolescência, cortando todos os laços com sua família e amigos. Quando emigrou, já apresentava antecedentes criminais por pequenos furtos para manter o uso de substâncias. Durante sua vida teve poucos encontros heterossexuais significativos e também referia ter tido relacionamentos homossexuais. Desde a adolescência o paciente referia apresentar fantasias e preferências sexuais por crianças (ambos os sexos, idades entre 6 e 9 anos), mas negava ter tido relações sexuais com essa população.

Aos 25 anos de idade foi admitido voluntariamente a um hospital psiquiátrico pela primeira vez por causa de um episódio depressivo e teve alta poucas semanas depois, com prescrição para inibidor seletivo da recaptação de serotonina (ISRS), que não utilizou. Após um mês foi novamente admitido, apresentando prodigalidade, impulsividade, jogo compulsivo e agitação psicomotora. Seis meses após a alta, aos 26 anos, apresentou uma tentativa de suicídio (pensava em se jogar do telhado de uma casa, mas foi detido pela polícia e por paramédicos) e foi novamente internado, com diagnóstico de episódio depressivo. Após alta com melhora do humor, o paciente abandonou o tratamento e passou a acessar regularmente pornografia infantil em cibercafés (fotos, vídeos, fóruns), sem preferência por sexo masculino ou feminino, sempre em locais diferentes, pagando em dinheiro para não ser identificado. Não teve qualquer relação sexual com adultos durante essa época. Após oito meses foi novamente internado, em novo episódio maníaco, para o qual foram prescritos clorpromazina, amitriptilina, fluoxetina e carbonato de lítio, e foi encaminhado para tratamento de manutenção com cloridrato de metadona e acompanhamento psiquiátrico em um centro ambulatorial de tratamento de toxicodependência.

\section{DISCUSSÃO}

A pedofilia, segundo o DSM-IV, é definida como intensa atração sexual, fantasias sexuais ou outros comportamentos de caráter sexual por pré-pubescentes, por um período de ao menos seis meses 5 . Nesse caso, o indivíduo com fantasias pedofílicas durante toda sua vida adulta, antes do início dos episódios maníacos ou depressivos, ilustra o caráter do pedófilo que apresenta intensas fantasias, mas não se engaja em atividades sexuais.

Pedófilos são geralmente homens imaturos emocionalmente, com diversos conflitos e sem a assertividade necessária para relações sexuais com mulheres maduras ${ }^{6}$, e que geralmente referem ter sido sexualmente abusados durante a infância? .

Embora a hipersexualidade seja um sintoma comum apresentado durante fases maníacas, parafilias são raramente relatadas na literatura. A dificuldade em descrever comportamentos parafílicos pode ser consequência do fato de que médicos não são informados da presença de comportamento sexual desviante, provavelmente pela vergonha em admitir esse comportamento. A hipersexualidade não justifica as fantasias pedófilas desse paciente, que as apresentou durante toda sua vida adulta, mas parece ter colaborado para a deterioração de seu comportamento, coincidindo com ativas buscas por pornografia infantil, comportamento não apresentado antes do início da doença.

Recentemente, levantou-se a hipótese de que a pedofilia pode ser enquadrada em transtornos do comportamento toxicodependente ${ }^{8}$. Tanto pedófilos quanto utilizadores de substâncias apresentam grau considerável de psicopatologia ${ }^{9-11}$, e a toxicodependência pode ser definida como uma condição caracterizada por falência recorrente em controlar um comportamento que, embora altamente recompensador a princípio, resulta em prejuízo significativo ${ }^{8}$, o que também parece ocorrer relativamente a comportamentos sexuais desviantes. Diversos estudos sugerem altos níveis de impulsividade em usuários de drogas ${ }^{10-13}$. O papel da impulsividade na pedofilia, entretanto, permanece controverso, e achados recentes sugerem que o transtorno pode ser classificado no espectro impulsivo-compulsivo ${ }^{8}$. Entre usuários de opioides que buscavam tratamento de manutenção com metadona, o transtorno de personalidade antissocial é a comorbidade psiquiátrica mais comumente encontrada ${ }^{13}$, e traços antissociais foram consistentemente encontrados

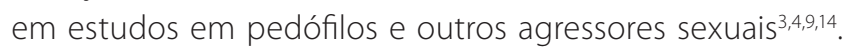
Elevados níveis de traços de personalidade antissocial em usuários de drogas também foram documentados por 
Craig $^{11}$ e Ball e Cecero $^{12}$, entre outros, mas a controvérsias sobre se esses traços são verdadeiras características de personalidade ou consequência dos efeitos diretos da droga e/ou do estilo de vida associado permanece ${ }^{13}$.

Em conclusão, o caso apresentado demonstra que, muito embora a hipersexualidade possa ser uma apresentação da fase maníaca no transtorno bipolar, as fantasias pedofílicas iniciaram antes do início do transtorno de humor, e a relação aparente entre eles parece ser o agravamento da busca compulsiva por pornografia infantil. A relação entre dependência química e transtornos de humor está bem estabelecida na literatura, mas são raros os relatos de caso de pacientes pedófilos dependentes químicos, embora diversos estudos demonstrem possível relação do espectro compulsivo entre os dois quadros. Cabe ao terapeuta, durante a entrevista clínica e a avaliação de risco (risk assessment), abordar questões sexuais relacionadas aos pacientes em tratamento para toxicodependência, avaliando possíveis inadequações e desvios e garantindo abordagem adequada de todas as comorbidades apresentadas.

\section{REFERÊNCIAS}

1. Ahlmeyer S, Kleinsasser D, Stoner J, Retzlaff P. Psychopathology of incarcerated sex offenders. J Pers Disord. 2003;17(4):306-18.
2. McElroy SL, Soutullo CA, Taylor Jr P, Nelson EB, Beckman DA, Brusman LA, et al. Psychiatric features of 36 men convicted of sexual offenses. J Clin Psychiatry. 1999;60(6):414-20.

3. Kafka MP, Prentky RA. Preliminary observations of DSM-II-R axis I comorbidity in men with paraphilias and paraphilia-related disorders. J Clin Psychiatry. 1994;55(11):481-7.

4. Raymond NC, Coleman E, Ohlerking F, Christenson GA, Miner M. Psychiatric comorbidity in pedophilic sex offenders. Am J Psychiatry. 1999;156(5):786-8.

5. American Psychiatric Association (APA). Diagnostic and statistical manual of mental disorders. 4th ed. Washington, DC: American Psychiatric Association; 1994.

6. Bridges MR, Wilson JS, Gacono CB. A Rorschach investigation of defensiveness, selfperception, interpersonal relations, and affective states in incarcerated pedophiles. J Pers Assess. 1998;70(2):365-85.

7. Freund K, Kuban M. The basis of the abused abuser theory of pedophilia: a further elaboration on an earlier study. Arch Sex Behav. 1994;23(5):553-63.

8. Stein DJ, Black DW, Pienaar W. Sexual disorders not otherwise specified: compulsive, addictive, or impulsive? CNS Spectr. 2000;5(1):60-4.

9. Cohen LJ, McGeoch PG, Watras-Gans S, Acker S, Poznansky 0, Cullen K, et al. Personality impairment in male pedophiles. J Clin Psychiatry. 2002;63(10):912-9.

10. Cohen LJ, Gertmenian-King E, Kunik L, Weaver C, London ED, Galynker I. Personality measures in former heroin users receiving methadone or in protracted abstinence from opiates. Acta Psychiatr Scand. 2005;112(2):149-58.

11. Craig RJ. Prevalence of personality disorders among cocaine and heroin addicts. Subst Abus. 2000;21(2):87-94.

12. Ball SA, Cecero JJ. Addicted patients with personality disorders: traits, schemas, and presenting problems. J Pers Disord. 2001;15(1):72-83.

13. Brooner RK, King VL, Kidorf M, Schmidt CW, Bigelow GE. Psychiatric and substance use comorbidity among treatment-seeking opioid addicts. Arch Gen Psychiatry. 1997;54(1):71-80.

14. Black DW, Kehrberg LLD, Flumerfelt DL, Schlosser SS. Characteristics of 36 subjects reporting compulsive sexual behavior. Am J Psychiatry. 1997;154(2):243-9. 